\title{
Self-organization, Pattern Formation, Cavity Solitons and Rogue Waves in Singly Resonant Optical Parametric Oscillators
}

\author{
Gian-Luca Oppo, ${ }^{*}$ Alison M. Yao, and Domenico Cuozzo \\ ICS, SUPA and Department of Physics, University of Strathclyde, Glasgow G4 ONG, Scotland, U.K.
}

\begin{abstract}
Spatio-temporal dynamics of singly resonant optical parametric oscillators with external seeding displays hexagonal, roll and honeycomb patterns, optical turbulence, rogue waves and cavity solitons. We derive appropriate mean-field equations with a $\operatorname{sinc}^{2}$ nonlinearity and demonstrate that offresonance seeding is necessary and responsible for the formation of complex spatial structures via selforganization. We compare this model with those derived close to the threshold of signal generation and find that back-conversion of signal and idler photons is responsible for multiple regions of spatio-temporal self-organization when increasing the power of the pump field.
\end{abstract}

PACS numbers: 42.50.Lc, 42.50.Dv, 42.65.Yj

Keywords: pattern formation, cavity soliton, optical parametric oscillator, turbulence, rogue waves

\section{INTRODUCTION.}

Transverse pattern formation, autosolitons and cavity solitons have been the subject of intense research in nonlinear optics in the last two decades since their original predictions [1-5]. Unlike in other fields of science, transverse patterns and dissipative solitons find useful applications in photonics such as optical memories, delay lines and optical registers [6]. Cavity solitons counterparts in the propagation direction have also been shown to generate passive mode-locking in fiber lasers [7].

Formation of transverse spatial structures in quadratic nonlinear cavities was predicted first in optical parametric oscillators (OPOs) $[8,9]$ and later extended to second harmonic generation [10, 11]. Early predictions in OPOs were confined to the degenerate case where signal and idler fields have the same frequency. Experimental evidence of pattern formation was indeed found in triply resonant degenerate OPOs close to the confocal cavity configuration [12] and via conical emissions [13, 14]. Confirmation of the predictions of [8] was provided in a broad-aperture degenerate OPOs in a plane-mirror minicavity [15]. Degenerate OPOs also display phase domain dynamics and dark-ring cavity solitons [17]. Finally, OPO models for non-degenerate Type-II cases in doubly or triply resonant cavity configurations have also been shown to display self-organization and pattern formation [16, 18-21].

Transverse instabilities in the case of non-degenerate, singly resonant OPOs (SROPOs), where the signal field is the only resonated field in an optical cavity, have been less discussed in the literature. On the theoretical side pattern formation in SROPOs is expected to replicate results of the complex Ginzburg-Landau laser case [18]. On the experimental side cw SROPO configurations are notoriously difficult to operate because of high oscillation thresholds (typically several watts) in common birefrin-

*Electronic address: g.1.oppo@strath.ac.uk gent crystals [22]. Quasi-phase matching in periodically poled materials has, however, considerably reduced operation thresholds of cw SROPOs [23] allowing for diode [24] and fiber [25] laser pumping for spectroscopy applications. A major advantage of cw SROPOs is that their wide tunability is monotonic and not affected by mode jumps typical of doubly or triply resonant configurations.

In this paper we investigate the formation and dynamics of transverse structures in SROPOs. We first derive a mean-field model in section 2 where the nonlinearity is of $\operatorname{sinc}^{2}$ form in agreement with early studies of SROPO steady states emissions [26-28]. The analysis builds on approaches that describe and integrate the propagation equations inside the OPO crystal $[29,30]$ by considering transverse effects and by carefully separating the meanfield and close-to-threshold approximations. The final model equations are capable of describing transverse pattern formation in the presence of pump depletion, signalidler recombination and external seeding close to the signal frequency. External seeding proves to be of fundamental importance for transverse structures in SROPOs since, in its absence, changes of the cavity length are compensated by changes in the signal (and idler) frequency thus nullifying the common mechanism of Turing pattern formation in off-resonant optical systems [1, 31].

In section 3 plane-wave steady states and their stability are analyzed in the SROPO models with external seeding, close to and far from threshold. These studies confirm that no pattern formation should be expected without a detuned external seed. Analytical expressions for the location in the parameter space of the loss of stability of homogeneous solutions to spatially modulated structures are then provided in section 4. The thresholds for pattern formation when changing the seeding intensity are then compared with those obtained from numerical integration of the SROPO dynamical equations with excellent agreement. Section 5 investigates when spatially periodic spatial structures break down to either optical turbulence for small seeding intensities or to cavity solitons for large pump and seeding intensities. Optical turbulence is demonstrated to be the mechanism 


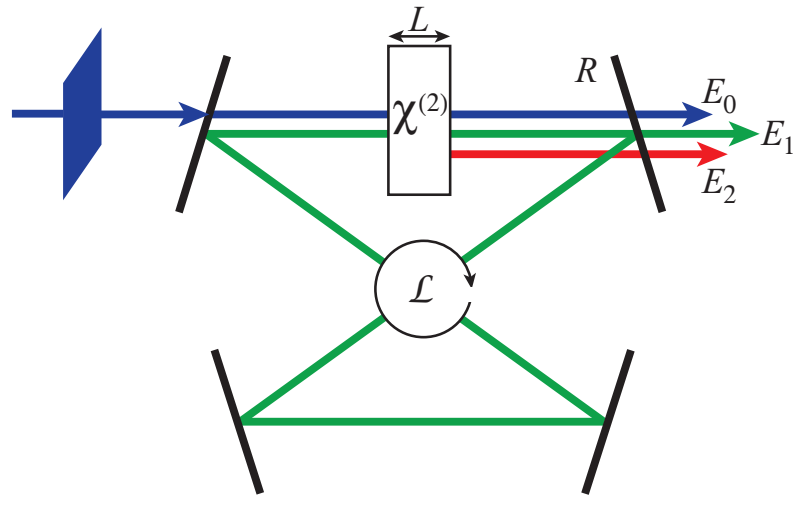

Figure 1: (Color online) Schematic diagram of a SROPO cavity of length $\mathcal{L}$ with a single partially reflecting mirror $R$ and containing a parametric down-conversion crystal of length $L$.

which generates rogue waves in the spatio-temporal evolution of the output fields. Finally, bright and dark cavity solitons are found in multistable configurations with localized hexagonal and honeycomb patterns.

\section{MEAN-FIELD MODELS.}

We consider parametric down conversion in a $\chi^{(2)}$ crystal of length $L$ at perfect phase matching, a condition that can also describe the average effect of quasi-phase matching in periodically poled crystals. In this case the propagation of the pump, signal and idler fields in the crystal along the $z$ direction are described by [32]:

$$
\begin{aligned}
& \partial_{z} E_{0}+\frac{n_{0}}{c} \partial_{t} E_{0}=\frac{i}{2 k_{0}} \nabla^{2} E_{0}-\alpha E_{1} E_{2} \\
& \partial_{z} E_{1}+\frac{n_{1}}{c} \partial_{t} E_{1}=\frac{i}{2 k_{1}} \nabla^{2} E_{1}+\mu \alpha E_{0} E_{2}^{*} \\
& \partial_{z} E_{2}+\frac{n_{2}}{c} \partial_{t} E_{2}=\frac{i}{2 k_{2}} \nabla^{2} E_{2}+\nu \alpha E_{0} E_{1}^{*}
\end{aligned}
$$

where $E_{j}$ with $j=0,1,2$ are the slowly varying amplitudes of pump, signal and idler fields, respectively, with wave-numbers $k_{j}=n_{j} \Omega_{j} / c$ and $\nabla^{2}$ is the transverse Laplacian operator along the $x$ and $y$ directions perpendicular to the propagation axis $z$. The frequency constraint $\Omega_{0}=\Omega_{1}+\Omega_{2}$ is rewritten as $\mu+\nu=1$ where $\Omega_{1}=\mu \Omega_{0}, \Omega_{2}=\nu \Omega_{0}$ and the effective coupling parameter $\alpha$ is given by

$$
\alpha=\frac{4 \pi \Omega_{0} \chi^{(2)}}{n c}
$$

where $\chi^{(2)}$ is the second order susceptibility of the crystal, $n=n_{0}=n_{1}=n_{2}$ is the common refractive index of the three waves that guarantees phase matching and $c$ is the speed of light in vacuum.

We assume that the parametric down conversion crystal is contained in an optical cavity of length $\mathcal{L}$ where the signal field is the only one to be resonated (see Figure 1). The steps involved in taking the mean-field approximation are the same as those reported in [21] although in the SROPO case there is only one resonated field. The final equation for the normalized signal field reads as:

$$
\begin{aligned}
\tau \partial_{t^{\prime}} E_{1} & +L \partial_{z} E_{1}=-\gamma E_{1}-i \delta E_{1}+i a \nabla^{2} E_{1} \\
& +\mu \alpha L E_{0} E_{2}^{*}+\sqrt{2 \gamma} E_{I N} .
\end{aligned}
$$

where we have introduced

$$
t^{\prime}=t-\left(\frac{\mathcal{L}-L}{c}\right) \frac{z}{L}
$$

and the parameters

$$
\begin{aligned}
\tau & =\frac{\mathcal{L}+(n-1) L}{c} ; & \gamma & =\frac{1-R}{2} ; \\
\delta & =\frac{\omega_{c}-\Omega_{1}}{c} \mathcal{L} ; & a & =\frac{\mathcal{L}}{2 k_{1}} .
\end{aligned}
$$

Here, $R$ is the output mirror reflectivity, $\omega_{c}$ is the frequency of the longitudinal cavity mode closest to the signal frequency $\Omega_{1}$ and $E_{I N}$ is a complex input field of frequency $\omega_{I N}$ close to $\Omega_{1}$, normally known as the seeding.

The usual mean-field limit procedure requires high reflectivity $R$ and involves an expansion in longitudinal Fourier modes and the requirement that all terms, including the nonlinear one, are independent of the longitudinal variable $z$. The $z$-variation per pass of the resonated signal field, $E_{1}$, can be neglected when it is affected by the average of the propagation of the pump and idler waves along the crystal [30], i.e.

$$
E_{1}=\frac{1}{L} \int_{0}^{L} E_{0}(z) E_{2}^{*}(z) d z
$$

To obtain an explicit dependence of pump and idler fields along the direction of propagation we consider the first and third equations of the system (1) and neglect diffraction in the crystal:

$$
\begin{aligned}
& d_{z} E_{0}(z)=-\alpha E_{1} E_{2}(z) \\
& d_{z} E_{2}(z)=\nu \alpha E_{0}(z) E_{1}^{*}
\end{aligned}
$$

where the signal amplitude $E_{1}$ is now independent of $z$. By taking the second derivative of (8) and using (9), one obtains

$$
d_{z}^{2} E_{0}(z)=-\left(\nu \alpha^{2} I_{1}\right) E_{0}(z)
$$

which shows that the pump field oscillates along the propagation direction with a frequency that depends on the signal intensity $I_{1}$. Integrating this equation we find

$$
E_{0}(z)=A_{0} \cos \left(\alpha \sqrt{\nu I_{1}} z\right)
$$

where $A_{0}$ is the amplitude of the pump field at the entrance of the crystal [30]. From (8),

$$
E_{2}(z)=-\frac{1}{\alpha E_{1}} d_{z} E_{0}=A_{0} E_{1}^{*} \sqrt{\frac{\nu}{I_{1}}} \sin \left(\alpha \sqrt{\nu I_{1}} z\right)
$$


in agreement again with [30].

We can now calculate the spatial average (7):

$$
\frac{1}{L} \int_{0}^{L} E_{0}(z) E_{2}^{*}(z) d z=\left|A_{0}\right|^{2} \frac{E_{1}}{2 \alpha L I_{1}} \sin ^{2}\left(\alpha L \sqrt{\nu I_{1}}\right)
$$

and insert it into (3):

$$
\begin{aligned}
\tau \partial_{t^{\prime}} E_{1} & +L \partial_{z} E_{1}=-\gamma E_{1}-i \delta E_{1}+i a \nabla^{2} E_{1} \\
& +\mu\left|A_{0}\right|^{2} \frac{E_{1}}{2 I_{1}} \sin ^{2}\left(\alpha L \sqrt{\nu I_{1}}\right)+\sqrt{2 \gamma} E_{I N}
\end{aligned}
$$

By expanding in longitudinal Fourier modes and retaining only the longitudinal mode closest to $\Omega_{1}$, corresponding to $\partial_{z^{\prime}} E_{1}=0$, we finally obtain:

$$
\begin{aligned}
\partial_{t^{\prime}} E_{1} & =\kappa\left[-(1+i \theta) E_{1}+i \hat{a} \nabla^{2} E_{1}\right. \\
& \left.+\mu\left|A_{0}\right|^{2} \frac{E_{1}}{2 \gamma I_{1}} \sin ^{2}\left(\alpha L \sqrt{\nu I_{1}}\right)+\hat{E}_{I N}\right]
\end{aligned}
$$

where

$$
\begin{aligned}
\kappa & =\frac{\gamma}{\tau}=\frac{\gamma c}{\mathcal{L}+(n-1) L} ; & \hat{a} & =\frac{a}{\gamma}=\frac{\mathcal{L}}{2 k_{1} \gamma} ; \\
\theta & =\frac{\delta}{\gamma}=\frac{\left(\omega_{c}-\Omega_{1}\right) \mathcal{L}}{c \gamma} ; & \hat{E}_{I N} & =\sqrt{\frac{2}{\gamma}} E_{I N} .
\end{aligned}
$$

Finally, we renormalize the transverse space variables $x$ and $y$ by dividing them by $\sqrt{\hat{a}}$, the time variable by multiplying it by $\kappa$ and the field amplitudes according to

$$
\begin{aligned}
E & =\alpha L \sqrt{\nu} E_{1} ; \quad\left|E_{0}\right|^{2}=\left|A_{0}\right|^{2} \frac{\mu \nu \alpha^{2} L^{2}}{2 \gamma} \\
E_{I N} & =\alpha L \sqrt{\nu} \hat{E}_{I N}
\end{aligned}
$$

to obtain

$$
\begin{aligned}
\partial_{\tau} E=\partial_{\kappa t^{\prime}} E & =E_{I N}-(1+i \theta) E \\
& +\left|E_{0}\right|^{2} \frac{E}{I} \sin ^{2}(\sqrt{I})+i \nabla^{2} E .
\end{aligned}
$$

The analysis of Eq. (18) is the main focus of the research presented here. It will be referred to as the $\sin c^{2}$ model since $\sin ^{2}(\sqrt{I}) / I=\operatorname{sinc}^{2}(\sqrt{I})$.

We note that in SROPO configurations the frequency of the signal field, $\Omega_{1}$, is tuneable by corresponding changes of the idler frequency, $\Omega_{2}$, while maintaining the energy conservation condition $\Omega_{0}=\Omega_{1}+\Omega_{2}$. This means that with no external seeding $\left(E_{I N}=0\right)$ the detuning $\theta$ is also zero since the SROPO tunes its signal frequency to the closest longitudinal cavity mode $\omega_{c}$. With an external seeding different from zero and detuned with respect to the cavity, it is advantageous to consider the external frequency $\omega_{I N}$ as reference and introduce

$$
\theta=\frac{\left(\omega_{I N}-\Omega_{1}\right) \mathcal{L}}{c \gamma} .
$$

Under these conditions $E_{I N}$ should be considered to be real and equation (18) remains unchanged.
It is interesting to investigate the behavior of the pump and idler fields inside the OPO crystal as provided by Eqs. (11) and (12). Figure 2 shows the pump and idler intensities during propagation for three sample values of $\left|E_{0}\right|^{2}$, namely $1.2,2.0$ and 8.0. While at $\left|E_{0}\right|^{2}=1.2$ (black lines) the changes of pump and idler per pass are limited, for $\left|E_{0}\right|^{2}=2.0$ (red lines) and $\left|E_{0}\right|^{2}=8.0$ (blue lines) they are substantial. In particular, full pump depletion and substantial back-conversion of signal and idler fields into the pump are clearly visible in Figure 2 for $\left|E_{0}\right|^{2}=8.0$. In the SROPO case these phenomena are not incompatible with the mean field approximation and are at the base of the sinc ${ }^{2}$ nonlinearity of model (18). The mean field approximation implies that the signal intensity remains almost constant with respect to its input value during propagation in the $\chi^{(2)}$ medium with large changes taking place over several cavity round-trips. No such constrains apply to pump and idler fields as shown in Fig. 2.

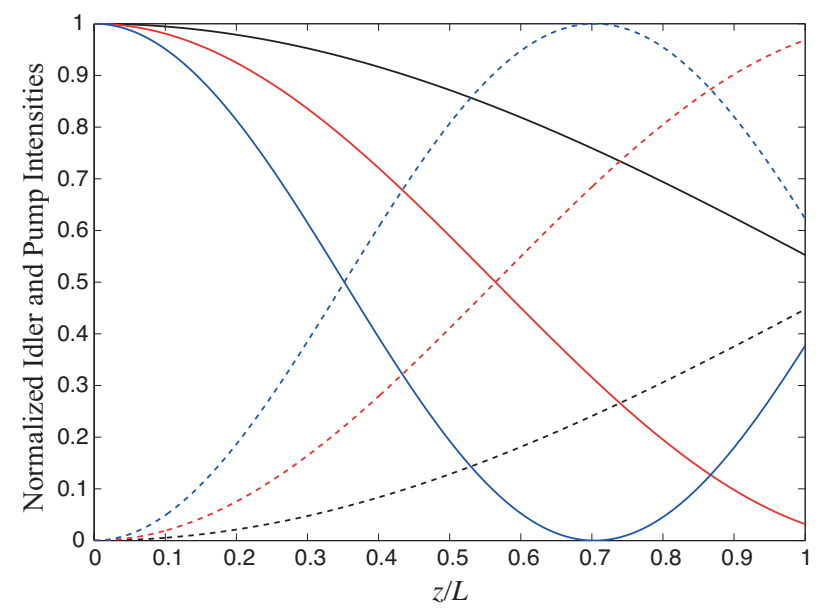

Figure 2: (Color online) Pump intensity (solid lines) and idler intensity (dashed lines) in the SROPO crystal for $\left|E_{0}\right|^{2}=1.2$ (black lines), $\left|E_{0}\right|^{2}=2.0$ (red lines) and $\left|E_{0}\right|^{2}=8.0$ (blue lines). The intensities are normalized to the input pump values $\left|E_{0}\right|^{2}$. The propagation distance is normalized to the crystal length.

Note that the $\cos ^{2}$ and $\sin ^{2}$ nature of the pump and idler intensities, respectively, guarantees conservation of the energy density in every point along the SROPO crystal. Energy conservation in turn guarantees the validity of the Manley-Rowe relations about the variations of the energy densities $N_{i}$ per field along the crystal:

$$
\frac{d N_{0}}{d z}=-\frac{d N_{1}}{d z}-\frac{d N_{2}}{d z}
$$

since $d N_{1} / d z=0$. These facts are a-posteriori confirmations that the physical processes described in Eqs. (1) are compatible with the application of the mean-field limit to the signal field even for large values of the pump and seeding intensities. 


\subsection{The close-to-threshold approximation.}

Close to the signal generation threshold it is possible to obtain partial differential equations in the mean-field limit where the nonlinear terms are in a polynomial form and thus easier to analyze. The scaling of the mean field limit requires that the nonlinear coefficient per pass, $\alpha L$, has to be of the order of the mirror transmittivity, $1-R$. This implies that the argument of the $\sin ^{2}$ term in equation (13) may become large for large signal intensities without breaking the mean-field conditions. Close to threshold, however, the signal intensity satisfies $I_{1}<1$ and the $\sin ^{2}$ term can be approximated by a power expansion. In this case pump and idler display small changes per pass across the crystal meaning that pump depletion and back-conversion do not take place in a single pass. Equations (11) and (12), however, tell us that while the pump can be approximated to first order to a constant value $A_{0}$, the idler has to grow along $z$ from its initial value. This is in agreement with previous analysis below threshold where the important noise term is associated with the idler fluctuations at the entrance of the crystal [33]. In the case of SROPOs close to threshold, we can approximate $E_{0}$ and $E_{2}$ in (11) and (12) with

$$
\begin{aligned}
& E_{0}(z) \approx A_{0}\left(1-\frac{\nu I_{1} \alpha^{2} z^{2}}{2}\right) \\
& E_{2}(z) \approx A_{0} E_{1}^{*}\left(\nu \alpha z-\frac{\nu^{2} I_{1} \alpha^{3} z^{3}}{6}\right) .
\end{aligned}
$$

By using these expressions to evaluate the average (7) one obtains:

$$
\begin{aligned}
& \frac{1}{L} \int_{0}^{L} E_{0}\left(z^{\prime}\right) E_{2}^{*}\left(z^{\prime}\right) d z^{\prime} \\
& \approx \frac{\nu \alpha L\left|A_{0}\right|^{2} E_{1}}{2}\left(1-\frac{\nu \alpha^{2} L^{2} I_{1}}{3}\right) .
\end{aligned}
$$

By repeating the same steps of the mean-field limit as described in the previous subsection we obtain:

$$
\partial_{\tau} E=E_{I N}-(1+i \theta) E+\left|E_{0}\right|^{2}\left(E-\frac{E I}{3}\right)+i \nabla^{2} E
$$

which describes the spatio-temporal behaviour of the SROPO close to threshold in the presence of an external seeding $E_{I N}$ and will be referred to as the cubic model.

\section{PLANE WAVE STEADY-STATES}

As mentioned in section 2, when there is no external seeding, $E_{I N}=0$, the detuning is zero since the SROPO automatically adjusts its frequency to the closest cavity resonance. The plane wave steady-state intensities, $I_{s}$, are implicit for the $\operatorname{sinc}^{2}$ model (see [26-28]) and explicit for the cubic model:

$$
\begin{aligned}
& I_{s}=\left|E_{0}\right|^{2} \sin ^{2}\left(\sqrt{I_{s}}\right) \\
& I_{s}=3\left(\left|E_{0}\right|^{2}-1\right) /\left|E_{0}\right|^{2}
\end{aligned}
$$

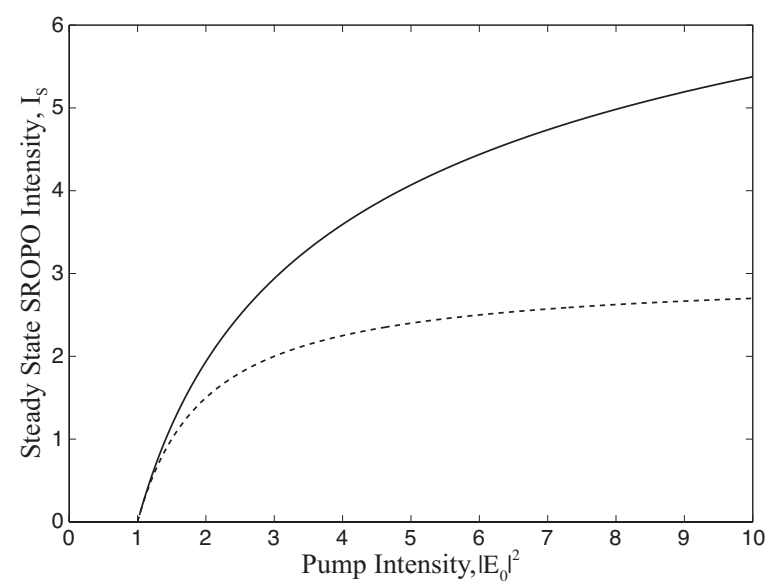

Figure 3: Intensity of the SROPO steady-state for the $\operatorname{sinc}^{2}$ model (solid line) and the cubic approximation (dashed line) with increasing pump intensities for $E_{I N}=0$ and $\theta=0$. All variables are dimensionless.

The steady-state signal intensity of the SROPO as a function of the pump intensity, $\left|E_{0}\right|^{2}$, is shown in Figure 3 for the $\operatorname{sinc}^{2}$ model (solid line) and the cubic approximation (dashed line). These are trivially complemented by the zero-intensity state that is stable below threshold, $\left|E_{0}\right|^{2}<1$, and unstable above. In the cubic case the stationary intensity above threshold asymptotes to the value 3 for large pump intensities and is always stable. The steady-state curve for the $\operatorname{sinc}^{2}$ model, on the other hand, becomes multivalued at large values of the input pump intensity $\left(\left|E_{0}\right|^{2}>20\right.$, not shown here) [27, 28]. Here, however, we are interested in values of the pump intensity below 10 , as these are more realistic with respect to present state-of-the-art of broad area SROPO realisations. In this regime it is possible to prove that, above threshold, the non-zero steady-state intensities in the sinc ${ }^{2}$ model are also stable [28]. Note that when comparing the $\operatorname{sinc}^{2}$ and the cubic models, there is a substantial difference between their steady-state intensities even below $\left|E_{0}\right|^{2}=2$. At twice above threshold this difference becomes considerable and the close to threshold (cubic) model has to be discarded.

Analogously to lasers, the field phase is decoupled from the steady-state equations and is affected by fluctuations and drift processes. When there is external seeding, $E_{I N}>0$, the phase of the SROPO locks to that of the external beam, depending on the magnitude of the detuning $\theta$ and the input intensity. Such behavior strongly differs from that of $E_{I N}=0$. In the case of $E_{I N} \neq 0$ the steady-state intensities are given by

$$
E_{I N}^{2}=I_{s}\left[\left(1-\left|E_{0}\right|^{2} f\left(I_{s}\right)\right)^{2}+\theta^{2}\right]
$$


where

$$
\begin{aligned}
& f\left(I_{s}\right)=\operatorname{sinc}^{2}\left(\sqrt{I_{s}}\right) \\
& f\left(I_{s}\right)=1-I_{s} / 3
\end{aligned}
$$

for the $\operatorname{sinc}^{2}$ and cubic models, respectively. The steadystate curves of the SROPO intensity versus the input intensity become S-shaped, a behavior typical of injected optical systems, as shown in section 4 .

For the cubic model without diffraction it is possible to obtain analytical results. For example, for $|\theta|<\left(\left|E_{0}\right|^{2}-\right.$ $1) / \sqrt{3}$ the plane-wave steady-state curves are S-shaped, and the positions of the turning points $\left[\left(E_{I N}^{2}\right)^{-}, I_{s}^{-}\right]$and $\left[\left(E_{I N}^{2}\right)^{+}, I_{s}^{+}\right]$can be determined by finding the maxima and minima of $(26)$ :

$$
I_{s}^{ \pm}=\frac{2\left(\left|E_{0}\right|^{2}-1\right) \pm\left(\left(\left|E_{0}\right|^{2}-1\right)^{2}-3 \theta^{2}\right)^{1 / 2}}{\left|E_{0}\right|^{2}}
$$

and then using these values in (26). At resonance, $\theta=0$, the turning points are located at:

$$
\begin{aligned}
{\left[\left(E_{I N}^{2}\right)^{+}, I_{s}^{+}\right]=} & {\left[0,3\left(\left|E_{0}\right|^{2}-1\right) /\left|E_{0}\right|^{2}\right] } \\
{\left[\left(E_{I N}^{2}\right)^{-}, I_{s}^{-}\right]=} & {\left[4\left(\left|E_{0}\right|^{2}-1\right)^{3} /\left(9\left|E_{0}\right|^{2}\right)\right.} \\
& \left.\left(\left|E_{0}\right|^{2}-1\right) /\left(3\left|E_{0}\right|^{2}\right)\right]
\end{aligned}
$$

Note that the + turning point at resonance corresponds to the zero seeding case of SROPO intensity given by Eq. (25).

\subsection{Linear stability analysis of the SROPO with seeding.}

The linear stability analysis of the steady-states given in the previous section produces two stability eigenvalues:

$$
\lambda_{ \pm}=\xi \pm \sqrt{\beta^{2}-\theta^{2}}
$$

where for the $\operatorname{sinc}^{2}$ model

$$
\begin{aligned}
& \xi=\left|E_{0}\right|^{2} \operatorname{sinc}\left(2 \sqrt{I_{s}}\right)-1 \\
& \beta=\left|E_{0}\right|^{2} \frac{\cos \left(2 \sqrt{I_{s}}\right)+\sqrt{I_{s}} \sin \left(2 \sqrt{I_{s}}\right)-1}{2 I_{s}}
\end{aligned}
$$

and for the cubic model

$$
\begin{aligned}
\xi & =\left|E_{0}\right|^{2}-1-2\left|E_{0}\right|^{2} I_{s} / 3 \\
\beta & =-\left|E_{0}\right|^{2} I_{s} / 3 .
\end{aligned}
$$

For the $\operatorname{sinc}^{2}$ model, the stability eigenvalues are implicit functions of the steady-state intensity, $I_{s}$. It is, however, easy to display the stability of the stationary states graphically along the S-shaped curves by picking increasing values of $I_{s}$, evaluating $\lambda_{ \pm}$and reporting the stability result on the diagram, as displayed in Figures 4 and 5.
Here black solid lines correspond to two negative real eigenvalues (sinks), turquoise solid lines to stable complex eigenvalues (foci), dot-dashed blue lines to at least one positive real eigenvalue (saddles or sources) and red dashed lines to complex eigenvalues with positive real part (unstable foci). In terms of bifurcations, the intersection of a black solid line and a blue dot-dashed line signals a saddle-node bifurcation, while the transition of a turquoise solid line into a red dashed line signals a Hopf bifurcation.

We find that the turning points of the S-shaped curves always correspond to either saddle-node (the $\left[\left(E_{I N}^{2}\right)^{+}, I_{s}^{+}\right]$points) or saddle-source (the $\left[\left(E_{I N}^{2}\right)^{-}, I_{s}^{-}\right]$ points) bifurcations corresponding to a change of sign of one real eigenvalue. For the cubic model this fact can be demonstrated analytically. In the lowest branch of the S-curve, the two real eigenvalues turn complex (see the red dashed line in Figures 4 and 5). This means that the lowermost part of the S-curve is Hopf unstable.

\section{TURING INSTABILITIES AND PATTERN FORMATION}

In this section we describe instabilities of the stationary states of the SROPO to transverse perturbations due to diffraction with and without external seeding. By moving to the spatial Fourier space of the transverse wave-vector $k$ and repeating the linear stability steps of the previous section, we obtain the two stability eigenvalues of (31) but with the detuning $\theta$ replaced by

$$
\theta_{k}=\theta+k^{2}
$$

which introduces an explicit dependence on the transverse spatial scale.

We start with the analysis of possible Turing instabilities without external seeding $\left(E_{I N}=0\right)$ and with zero detuning $\theta=0$. In this case, the evaluation of the stability eigenvalues with the appropriate factor (35) is done only at the values of $I_{s}$ given by (25). In the cubic case the eigenvalues reduce to:

$$
\lambda_{ \pm}=-\left(\left|E_{0}\right|^{2}-1\right) \pm \sqrt{\left(\left|E_{0}\right|^{2}-1\right)^{2}-k^{4}} .
$$

The largest eigenvalue has a zero value for the plane-wave case, $k=0$, corresponding to the uncoupled phase of the SROPO models without seeding as studied in the previous section. For large wave-vectors the eigenvalues can become complex, i.e. one may observe damped oscillations. However, the presence of diffraction cannot make the real part of the eigenvalues positive which means that, for the SROPO alone, there are no spatio-temporal instabilities and hence no pattern formation. We obtain the same result for the sinc ${ }^{2}$ model within the pump intensity ranges studied here although the implicit nature of the steady-state (25) requires straightforward numerical evaluations of the stability eigenvalues for given wavevectors $k$. 

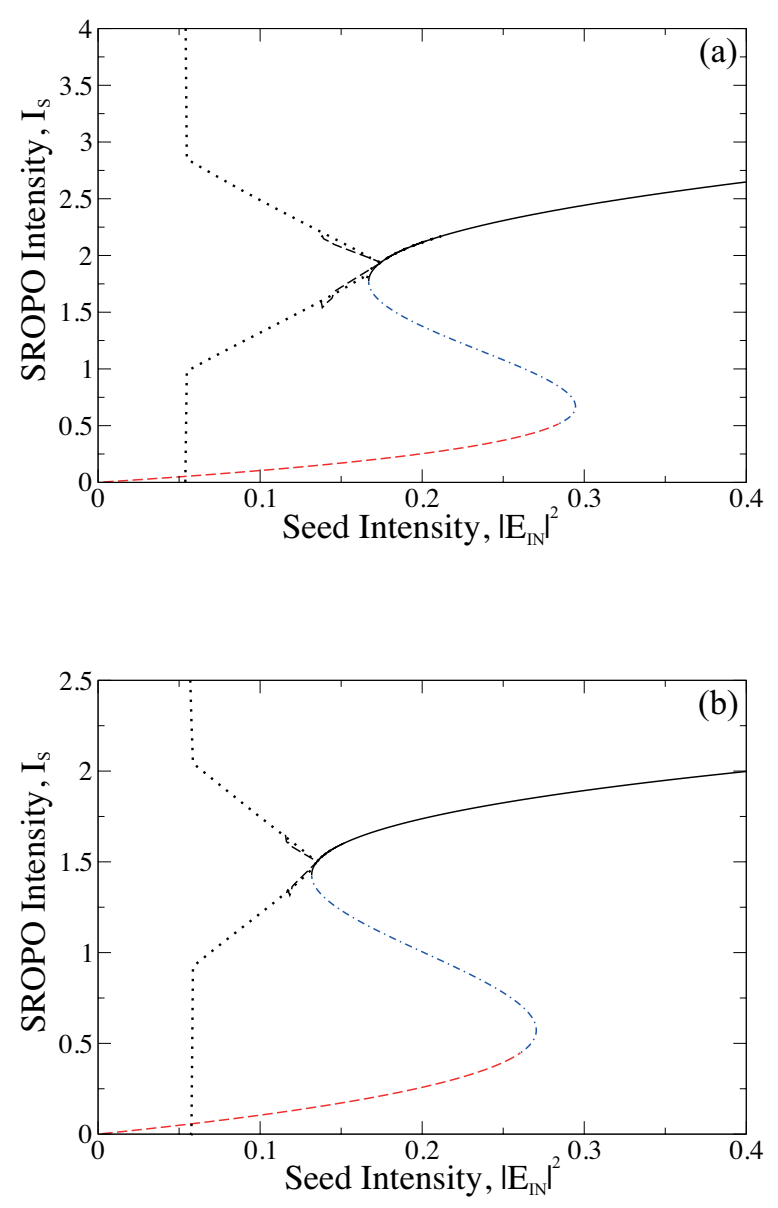

Figure 4: (Color online) Plane wave steady-state stability and pattern formation for (a) the $\operatorname{sinc}^{2}$ model (18) of a SROPO and (b) the cubic model (24) of a SROPO close to threshold. The solid (black), dot-dashed (blue) and dashed (red) lines correspond to stable, unstable and Hopf unstable plane wave steady states, respectively. The black dotted (black dashed) lines correspond to the minimum and maximum of the intensity of stationary hexagonal (roll) patterns. The vertical dotted line corresponds to the instability of hexagons leading to optical turbulence. Parameters are $\left|E_{0}\right|^{2}=2$ and $\theta=-0.3$. All variables are dimensionless.

We now consider the case of external seeding where the detuning, $\theta$, can be non-zero. By using the expressions (31) with $\theta$ replaced by $\theta_{k}$ (35) one observes that the transverse wave-vector can destabilise the system only when it counterbalances the detuning and that this is most effective when

$$
k^{2}=-\theta
$$

i.e. the off-resonance mechanism for pattern formation typical of optical systems $[1,8]$. We refer to the offresonance mechanism as Turing pattern formation since it has been demonstrated that all the requirements of Turing instabilities are fully satisfied [31].
The condition (37) provides us with the value along the steady-state curves at which we expect pattern formation to occur, $I_{s}^{c}$. This value simply corresponds to the steady-state value of the plane wave solution at zero detuning (25) since for $\theta_{k}=0$ the stability eigenvalues (31) reduce to $\lambda_{ \pm}=\xi \pm \beta$, where $\xi$ and $\beta$ are given by (32) for the sinc ${ }^{2}$ model and (34) for the cubic model. By tracing a horizontal line at the $I_{s}^{c}$ value on the diagrams of Figures 4 and 5 one obtains the corresponding value of $\left|E_{i n}^{c}\right|^{2}$ of the seeding intensity where the Turing instability takes place. The bifurcation from the homogeneous states to steady transverse patterns is obtained when decreasing the seeded amplitude $E_{I N}$ so that the locked plane wave state progressively approaches the upper turning point of the S-shaped steady-state curve (the $\left[\left(E_{I N}^{2}\right)^{+}, I_{s}^{+}\right]$point $)$. Before reaching it, the stationary plane wave intensity reaches the value $I_{s}^{c}$ and a stationary roll pattern is formed supercritically while a hexagonal pattern is formed subcritically in agreement with [35]. This bifurcation scenario is in agreement with early analysis of complex Ginzburg-Landau models in the presence of injection [36, 37]) although our cubic model does not contain diffusion or purely imaginary nonlinearities. It is also in remarkable agreement with numerical simulations, as demonstrated in section 4.1 .

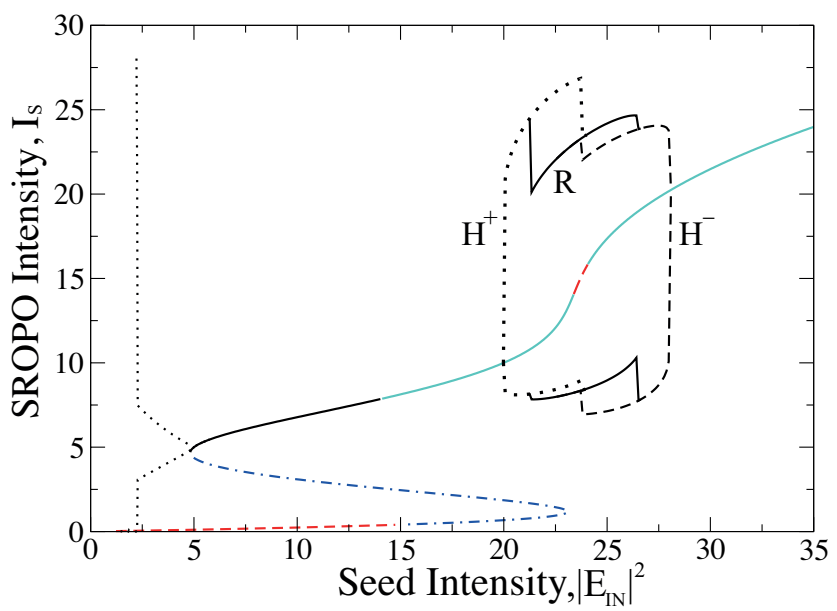

Figure 5: (Color online) Same as Fig. 4 but for parameter values $\left|E_{0}\right|^{2}=8$ and $\theta=-1$ (all variables are dimensionless). The solid turquoise lines correspond to stable plane wave steady states with complex stability eigenvalues. For seed intensities above 20, minima and maxima of the intensity of stable hexagonal patterns $\mathrm{H}^{+}$(dotted lines), of stable roll patterns R (solid lines) and of stable honeycomb patterns $\mathrm{H}^{-}$(dashed lines) are displayed.

We have also investigated instabilities of the plane wave to pattern structures for large values of both the input pump and the seeding intensity as shown in Fig. 


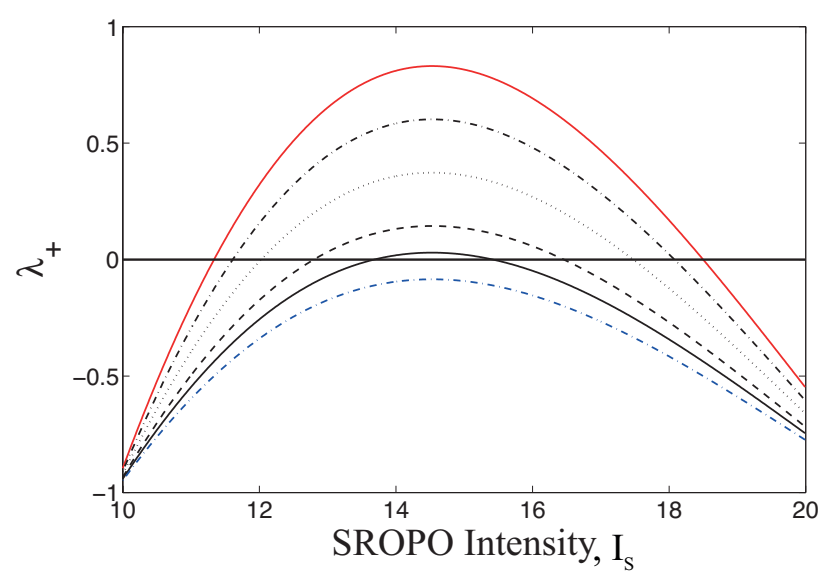

Figure 6: (Color online) Stability eigenvalue $\lambda_{+}$versus the SROPO stationary intensity. Parameters are $\theta=-1,\left|E_{0}\right|^{2}=$ 4 (lowermost dashed-dotted blue line), $\left|E_{0}\right|^{2}=4.5$ (solid black line), $\left|E_{0}\right|^{2}=5$ (dashed black line), $\left|E_{0}\right|^{2}=6$ (dotted black line), $\left|E_{0}\right|^{2}=7$ (uppermost dashed-dotted black line) and $\left|E_{0}\right|^{2}=8$ (uppermost solid red line). All variables are dimensionless.

5. These instabilities have no counterpart in the close-tothreshold regime and can be estimated analytically by using the stability eigenvalues (31) with (32) and $\theta_{k}=0$ for the most unstable wave-vector (37). Figure 6 shows the instability eigenvalue $\lambda_{+}$versus the stationary SROPO intensity for different values of the input pump $\left|E_{0}\right|^{2}$. Above a threshold value of $\left|E_{0}\right|^{2} \sim 4.37$ (corresponding to a critical value of $I_{s}=14.5$ ), there is a range of values of the SROPO intensity where the plane wave solution is unstable to spatial patterns. The limit values of the SROPO intensity are the zeroes of the $\lambda_{+}$curve shown in Figure 6 with the lower (upper) intersection corresponding to an instability when increasing (decreasing) the seeding intensity. In Figure 7 we show the plane-wave instability range in the parameter space of the SROPO intensity versus the seed intensity for different values of the pump intensity. In section 4.1 we show that the bifurcations at the boundaries of the instability ranges are subcritical in nature and that there are extended regions of bistability between patterns and stable plane waves to support cavity solitons. The ranges displayed in Fig. 7 provide a minimum size of the parameter region where pattern formation is expected. For example, the planewave instability range for $\left|E_{0}\right|^{2}=8$ evaluated analytically from the stability eigenvalues is approximately between $\left|E_{I N}\right|^{2}=22$ and $\left|E_{I N}\right|^{2}=26$ (see Fig. 7) while the numerical simulations find stable patterns between $\left|E_{I N}\right|^{2}=20$ and $\left|E_{I N}\right|^{2}=28$ because of subcriticality (see Fig. 5).

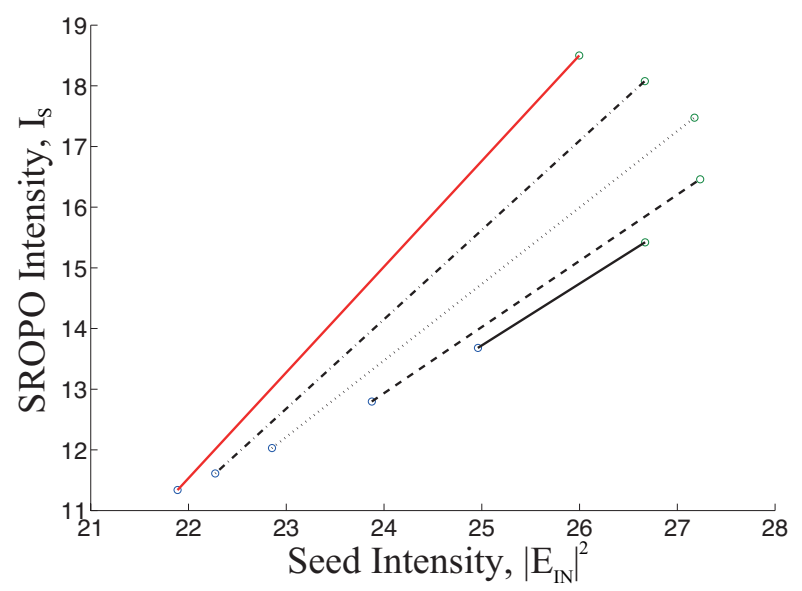

Figure 7: (Color online) Plane wave instability regions to spatial patterns in the (seed intensity, SROPO intensity) parameter space. Parameters are $\theta=-1,\left|E_{0}\right|^{2}=4.5$ (solid black line), $\left|E_{0}\right|^{2}=5$ (dashed black line), $\left|E_{0}\right|^{2}=6$ (dotted black line), $\left|E_{0}\right|^{2}=7$ (uppermost dashed-dotted black line) and $\left|E_{0}\right|^{2}=8$ (uppermost solid red line). All variables are dimensionless.

\subsection{Numerical patterns}

We have first numerically integrated the $\operatorname{sinc}^{2}(18)$ and cubic (24) models for $\left|E_{0}\right|^{2}=2$ and $\theta=-0.3$. We have started with relatively large values of the seeding amplitude, $E_{I N}=0.45$, where the stable plane-wave solution has been recovered. By progressively decreasing $E_{I N}$, a supercritical roll pattern is observed to appear at around $E_{I N}=0.424, I_{s}^{c}=1.9$ for the $\operatorname{sinc}^{2}$ model and $E_{I N}=0.374, I_{s}^{c}=1.5$ for the cubic model, in excellent agreement with the theoretical predictions given in section 4. By further decreasing the seeding intensity, the amplitude of the roll pattern increases (see black dashed lines in Fig. 4 until it merges into a hexagonal structure. Having located the hexagonal pattern (see Fig. 8 (a) for its transverse intensity structure), we have traced it with increasing and decreasing values of the external seeding intensity. For small seeding intensities, Figures 4 and 5 show the maximum and minimum intensity of the hexagonal pattern (dotted lines) and show that these change linearly with decreasing seeding intensity. The bifurcation back to the steady plane-wave solution is subcritical although the regime of sub-critical bistability is very small and difficult to detect on the scales of the diagrams. When further decreasing the external seeding, one observes a sudden destabilisation of the hexagonal pattern into a region of optical turbulence. The abrupt transition from stable patterns to turbulence is clearly displayed in Figures 4 and 5 by the almost vertical line on the right hand side of these diagrams that corresponds to a sudden jump in the values of the minima and maxima intensities observed in the transverse section during the turbulent evolution. 

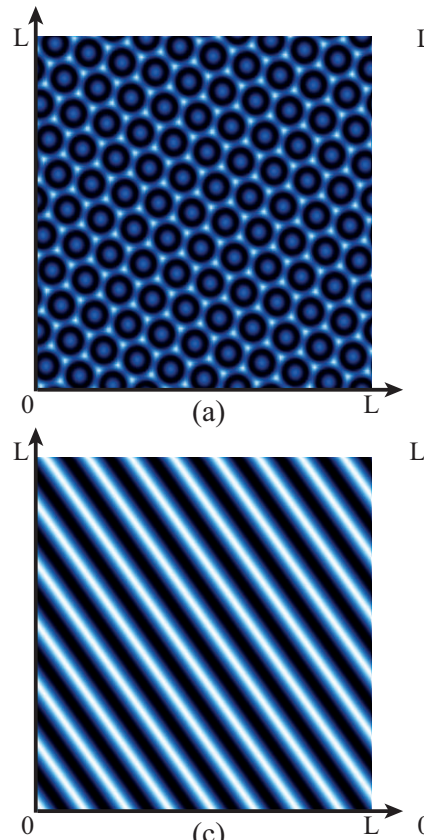

(c)
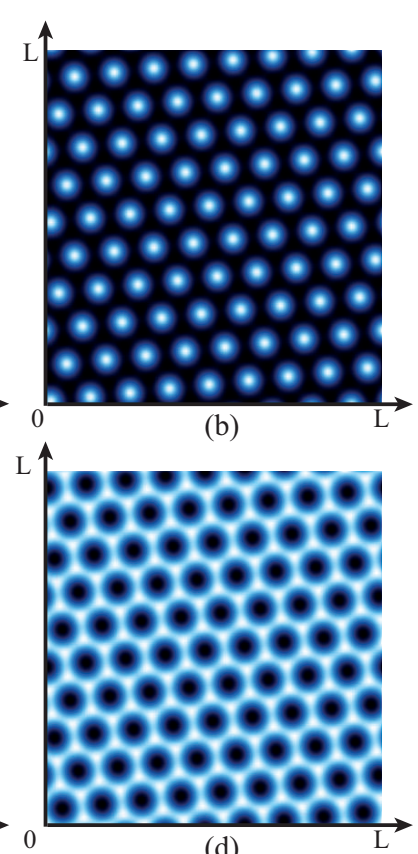

(d)

Figure 8: (Color online) Intensity of transverse patterns in a SROPO. (a) Hexagons for $\left|E_{I N}\right|^{2}=3$. (b) Hexagons for $\left|E_{I N}\right|^{2}=22$. (c) Rolls for $\left|E_{I N}\right|^{2}=24$. (d) Honeycombs for $\left|E_{I N}\right|^{2}=27$. Parameters are $\left|E_{0}\right|^{2}=8$ and $\theta=-1$. All variables are dimensionless.

For larger values of the input pump, $\left|E_{0}\right|^{2}$, new regions of pattern formation arise in the SROPO with seeding in a way similar to what has been described for nascent optical bistability [4]. These new regions can only be observed in the sinc ${ }^{2}$ model since the cubic model can only display stable plane wave solutions for large $\left|E_{0}\right|^{2}$ and large $E_{I N}^{2}$. Moreover, the cubic model is not accurate away from threshold. For the numerical simulations presented here we have selected the value of $\left|E_{0}\right|^{2}=8$ where the minimum size of the pattern region is more than $15 \%$ of the maximum value of the seed intensity in order to guarantee relevance to possible experimental realizations. In Fig. 5 we present the intensities of the observed patterns together with the steady-state plane wave curves for the selected value of $\left|E_{0}\right|^{2}=8$. At low seed intensities the phenomenology is similar to that described for $\left|E_{0}\right|^{2}=2$ above. However, at larger seeding intensities the upper branch of the S-shaped plane-wave steady-state curve suddenly increases. The steady-state first develops damped oscillations and then becomes unstable to a Hopf bifurcation (see dashed red lines around the seed intensity of 23 in Figure 5). Around such bifurcation, a new region of stationary patterns develops. We have identified rolls $\mathrm{R}$ (solid lines), hexagons $\mathrm{H}^{+}$(dotted lines) and honeycombs $\mathrm{H}^{-}$(dashed lines). The intensities of the different transverse patterns are displayed in Figure 8. We note that none of the patterns observed at large input pumps and seeding intensities are present in the cubic model. Finally, pattern bistability is observed

between rolls and hexagons and rolls and honeycombs.

\section{OPTICAL TURBULENCE, ROGUE WAVES AND CAVITY SOLITONS.}

When the seeding is small, the input energy is not sufficient to lock the SROPO to the external laser. These unlocked regimes are typical of lasers with injected signals [38]. The larger the detuning, $\theta$, between the external laser and the SROPO cavity, the larger the seed intensity necessary for locking. Since the lower branch of the S-shaped steady-state curves is always Hopf unstable for small seeding, one expects to observe dynamical regimes where locking and unlocking alternate in space and time. In comparison with purely temporal systems, the presence of transverse degrees of freedom elongates the locking region to lower values of the seeding intensity, as displayed in Figures 4 and 5 where stable hexagons are observed well into the region where plane wave solutions are unstable. As the seeding intensity is decreased, unlocking eventually takes place and stable patterns develop defects $[37,39]$ that induce first phase and then amplitude instabilities. The resulting regime corresponds to optical turbulence since one observes a sudden (exponential) decrease of the spatio-temporal correlation function [40]

$$
C(\rho)=\frac{\operatorname{Re}\left[\left\langle E(\mathbf{r}, t) E^{*}\left(\mathbf{r}^{\prime}, t\right)\right\rangle-\langle E(\mathbf{r}, t)\rangle\left\langle E^{*}(\mathbf{r}, t)\right\rangle\right]}{\operatorname{Re}\left[\left\langle E(\mathbf{r}, t) E^{*}(\mathbf{r}, t)\right\rangle-\langle E(\mathbf{r}, t)\rangle\left\langle E^{*}(\mathbf{r}, t)\right\rangle\right]}
$$

where $\mathbf{r}$ and $\mathbf{r}^{\prime}$ identify separate positions on the transverse plane, $\rho=\left|\mathbf{r}-\mathbf{r}^{\prime}\right|$, Re denotes the real part and $\langle\cdot\rangle$ corresponds to temporal averages. Such behavior is demonstrated in Figure 9 where the correlation function $C(\rho)$ is calculated for the hexagonal pattern (dashed line), the turbulent regimes for $\left|E_{0}\right|^{2}=2$ (solid line) and $\left|E_{0}\right|^{2}=8$ (dot-dashed line). Fitting exponentials to the correlation functions shows that in the turbulent regimes the correlation length is reduced by at least a factor of six. In the regime of optical turbulence, large variations of the SROPO intensity are observed in both space and time. In Figures 4 and 5 we display the range of variation of the SROPO intensity at a given time $t$ at the onset of optical turbulence. The wide increase in the maximum SROPO intensity when changing the seed strength below the hexagon instability is clearly visible.

To characterize the regime of optical turbulence we have considered the temporal evolutions of the maximum SROPO intensity, the spatial average of the SROPO intensity and its standard deviation. As displayed in Figure 10, the spatial statistics is large enough to guarantee probability distributions of well defined averages and deviations. Larger values of the pump power increase the size of the probability distribution of the SROPO intensity and that of the fluctuations of its maximum value (compare Figures 10(a) and (b)). Such increase results in the occurrence and propagation of transverse rogue waves. 


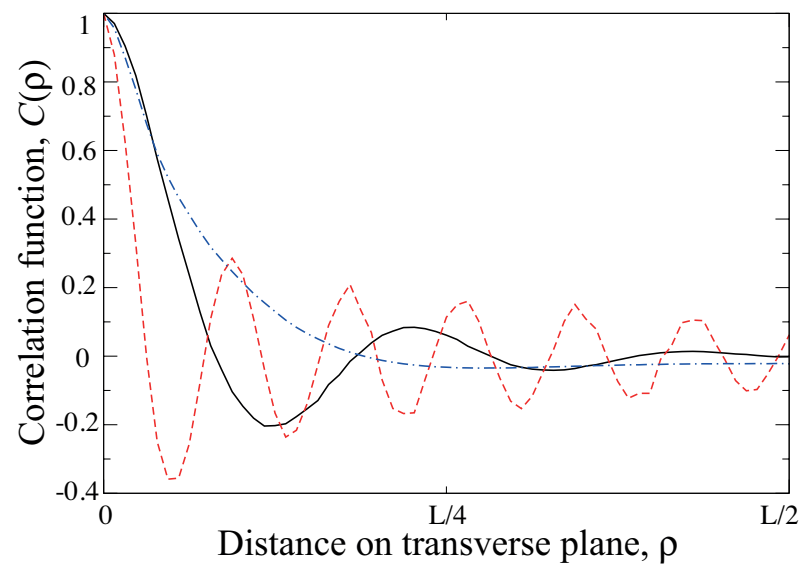

Figure 9: (Color online) Spatial correlation function $C(\rho)$ as in (38) for the hexagonal pattern (red dashed line, $\left|E_{0}\right|^{2}=2$, $\left.\theta=-0.3,\left|E_{I N}\right|^{2}=0.09\right)$, optical turbulence close to threshold (black solid line, $\left|E_{0}\right|^{2}=2, \theta=-0.3,\left|E_{I N}\right|^{2}=0.04$ ) and away from threshold (blue dot-dashed line, $\left|E_{0}\right|^{2}=8$, $\left.\theta=-1.0,\left|E_{I N}\right|^{2}=2.19\right)$. All variables are dimensionless.

Following the generally accepted definition of rogue waves in systems with injection [41], we plot the temporal evolution of

$$
q(\tau)=I_{x, y}^{M a x}(\tau)-\left\langle\langle I\rangle_{x, y}\right\rangle_{\tau}-8\left\langle\langle\sigma\rangle_{x, y}\right\rangle_{\tau}
$$

corresponding to transverse pulse maxima, $I_{x, y}^{M a x}$, above or below a threshold given by the average value of the intensity, $\langle I\rangle_{x, y}$, plus eight times the standard deviation, $\sigma_{\tau, x, y}$, of the SROPO intensity for the $\operatorname{sinc}^{2}$ model in the dashed-dotted red lines of Figures 10(a) and (b). The presence of peaks of a rogue wave is signalled by positive values of $q(\tau)$ [41]. With pump intensities a few times above threshold (Figure 10(a)), the rogue wave test fails $(q(\tau)$ remains negative) and the optical turbulence generated by the unlocking of the seed laser and the SROPO is relatively mild. With larger values of the pump power, however, rogue waves are commonplace and affect the spatio-temporal evolution of the SROPO field for long durations of the temporal evolution (see Figure 10(b)). When comparing these results with those related to lasers with injections [41], we note that our simulations are fully spatio-temporal and show that the material dynamics, typical of semiconductor media, is not essential in the generation and maintenance of rogue waves during optical turbulence. The main mechanism underlying rogue waves in SROPOs is the absence of locking between master and slave devices leading to intermittent phase jumps. Full investigations of optical turbulence in injected (seeded) optical devices will be presented elsewhere.

Finally, we have studied the presence and stability of cavity solitons (CS) in SROPOs with a particular focus
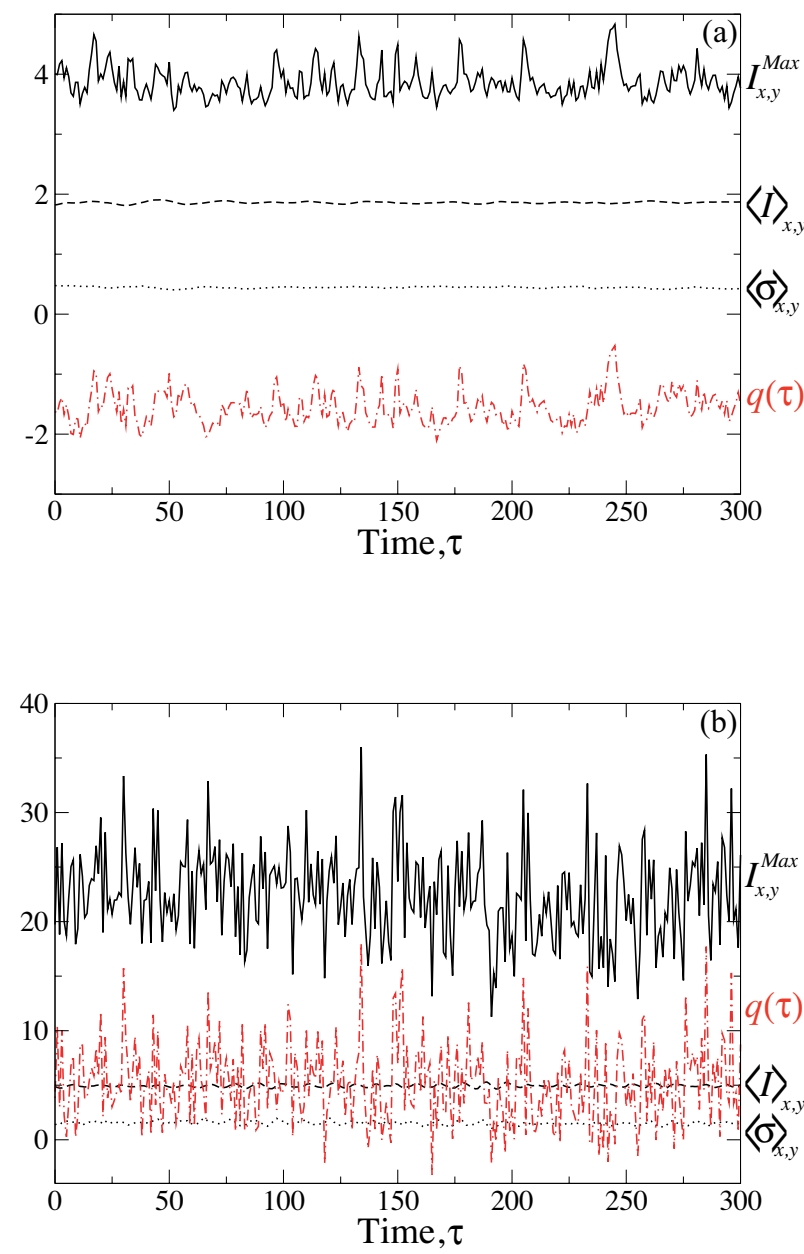

Figure 10: (Color online) Temporal evolution of the maximum SROPO intensity (solid black line), the spatially averaged SROPO intensity (dashed black line), its standard deviation (dotted black line) and $q(\tau)$ for the sinc ${ }^{2}$ model (18). Parameters are (a) $\left|E_{0}\right|^{2}=2, \theta=-0.3,\left|E_{I N}\right|^{2}=0.04$ and (b) $\left|E_{0}\right|^{2}=8, \theta=-1,\left|E_{I N}\right|^{2}=2.19$. All variables are dimensionless.

on localised structures induced by the $\operatorname{sinc}^{2}$ nonlinearity, i.e. away from threshold and with large seeding from an external laser. CS have been described in a variety of OPO devices without seeding from degenerate [17, 42-44] to non-degenerate triply resonant configurations $[20,45$, 46]. CS in degenerate OPOs have also been numerically extended to include the presence of seeding [47]. In the case of the non-degenerate SROPOs investigated here, the resonance condition of SROPO operation rules out any CS in the absence of seeding. It is then important to stress that all CS solutions described in this section are due to the external seeding field and have no counterpart 
in the case of $E_{I N}=0$.

Since we have introduced the $\operatorname{sinc}^{2}$ nonlinearity in spatio-temporal models of SROPOs to describe selforganization when pump depletion and back-conversion take place, we focus here on CS in the limit of large pump powers. From Figures 5 and 7 we see that there are broad ranges of the parameter space where bistability between the plane wave solution and pattern structures is observed. For example, we find coexistent hexagons and homogeneous solutions for $\left|E_{I N}\right|^{2}$ between 19.98 and 21.90 and coexistent honeycombs and homogeneous solutions for $\left|E_{I N}\right|^{2}$ between 26.00 and 28.09. Note that we even observe tri-stability among plane waves, hexagons and rolls for $\left|E_{I N}\right|^{2}$ between 21.25 and 21.90 and among plane waves, honeycombs and rolls for $\left|E_{I N}\right|^{2}$ between 26.00 and 26.52. In the two wide regions of homogeneouspattern bistability we have been able to locate single peak (bright) and single trough (dark) CS as shown for example in Figure 11 (a) and (d), respectively. The onset and nature of these CS are again similar to those observed in nascent optical bistability [4]. Together with the single unit bright and dark CS we have also found many multi-peak [48] and multi-trough localized structures that correspond to clusters of CS (also referred to as localized patterns [49]). A few examples of these bright and dark clusters are displayed in Figure 11. The range of existence of single unit CS and CS clusters is displayed in Figure 12. Snaking of both bright and dark CS is observed with stability branches of larger and larger clusters approaching the pattern stability lines in the parameter space (see Figure 12). The details of the bifurcations and of the number of branches of bright and dark CS for changing $\left|E_{0}\right|^{2}$ are too long to be described here and will be the subject of a future publication.

\section{CONCLUSIONS.}

Self-organization and pattern formation in OPOs has been known for a number of years in degenerate [8] or doubly or triply resonant non-degenerate configurations [19-21]. The case of a widely non-degenerate SROPO has, however, been overlooked because of experimental limitations, now overcome, and the fact that offresonance operation is inhibited because of its intrinsic tuneability. Here we have shown that under the action of a detuned injection close to the signal frequency, one can find an extremely rich variety of self-organized structures, from regular co-existing patterns to clusters of CS and even optical turbulence. In particular, we have derived mean field models for SROPOs with external seeding and shown that, away from threshold, cubic nonlinearities should be replaced by $\operatorname{sinc}^{2}$ terms. The $\operatorname{sinc}^{2}$ nonlinearity is capable of describing regimes of pump depletion and back-conversion. In these regimes, the external seeding generates hexagonal, roll and honeycomb patterns as well as bright and dark CS. Note that CS in SROPOs offer positional control associated to the generation of
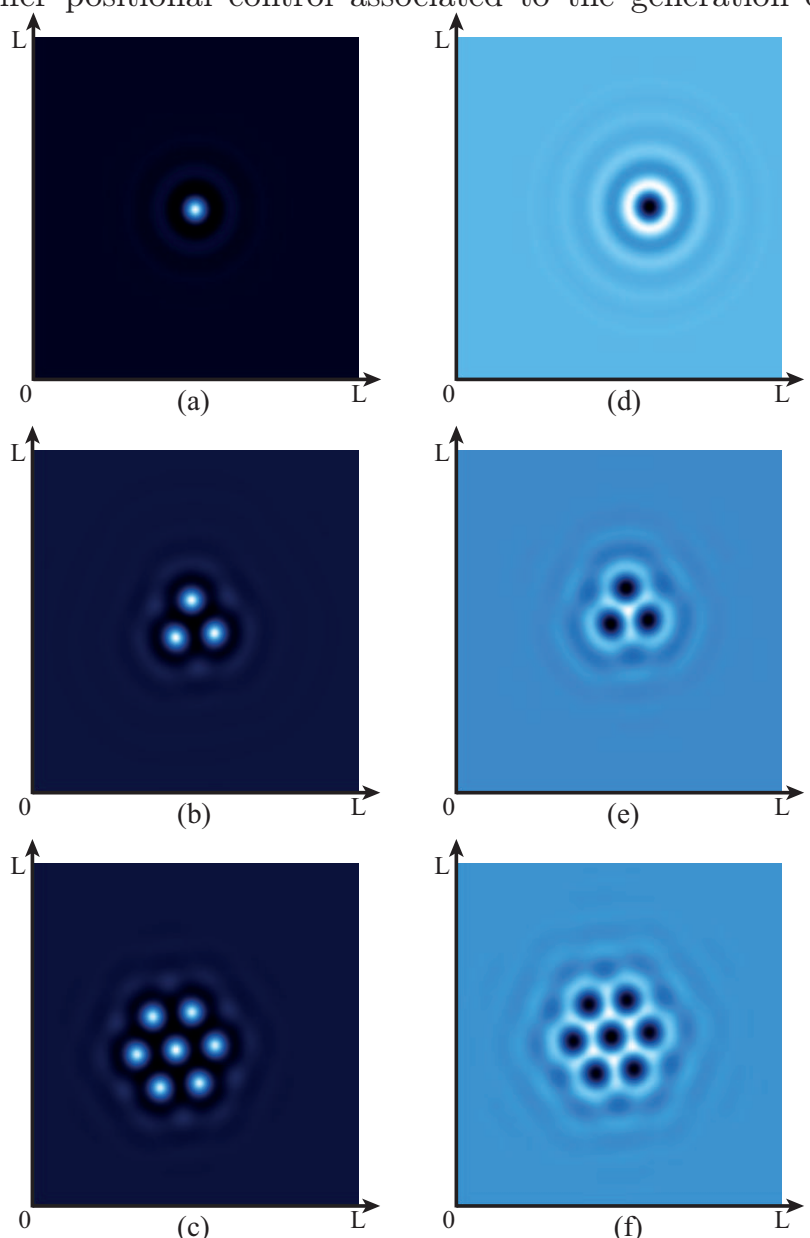

Figure 11: (Color online) Stable bright (a)-(c) and dark (d)(f) CS configurations of the SROPO model (18). Parameters are $\left|E_{0}\right|^{2}=8, \theta=-1,\left|E_{I N}\right|^{2}=20.8$ for (a)-(c), $\left|E_{I N}\right|^{2}=$ 26.5 for (d) and $\left|E_{I N}\right|^{2}=27.1$ for (e)-(f). All variables are dimensionless.

entangled photons with vastly different frequencies.

In contrast to laser systems, the fast material dynamics of $\chi^{(2)}$ media makes a SROPO with external seeding an ideal candidate for comparisons between theory and experiments of optical self-organization. The fast material dynamics is also beneficial to the investigation of spatio-temporal structures in the regime of short pulse generation where many of the results presented here can find useful extensions. These investigations together with the full characterization of the turbulent regimes will be the subject of future communications.

\section{Acknowledgments.}

We thank J. Lega for useful discussions. We acknowledge financial support from the EU grant HIDEAS. AMY acknowledges financial support from the UK Engineering and Physical Sciences Research Council (EPSRC). 


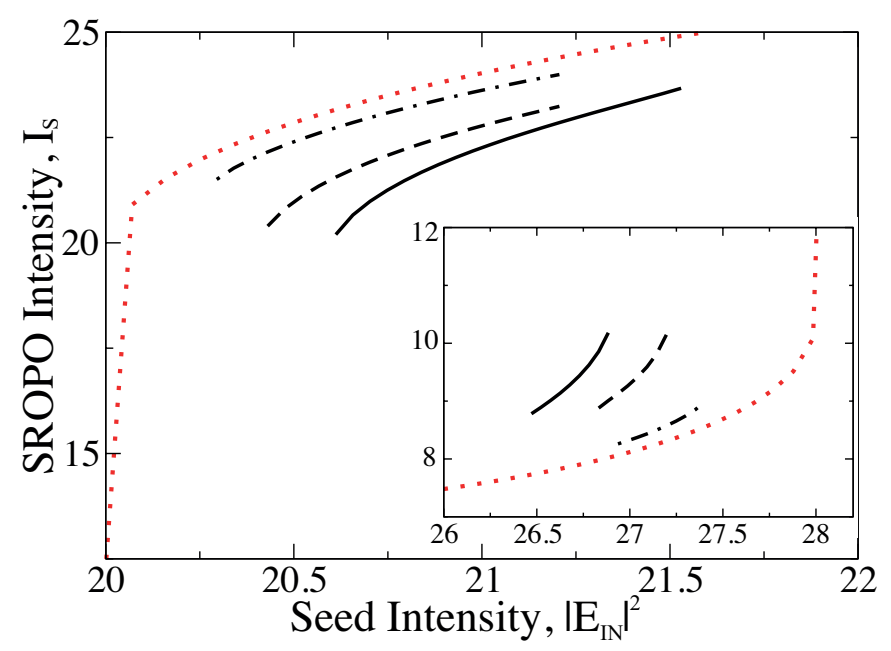

Figure 12: (Color online) Stability range of clusters of CS for bright (main figure) and dark (inset) CS. Solid, dashed and dot-dashed black lines correspond to the maximum SROPO intensity in clusters of one, three and seven CS. The red dotted line corresponds to the maximum (minimum) intensity of the stable hexagonal (honeycomb) pattern in the main figure (inset). Parameters are $\left|E_{0}\right|^{2}=8$ and $\theta=-1$. All variables are dimensionless.

[1] L. A. Lugiato and R. Lefever, Phys. Rev. Lett. 58, 2209 (1987)

[2] G. D'Alessandro and W. J. Firth, Phys. Rev. Lett. 66, 2597 (1991)

[3] N. N. Rosanov, A. V. Fedorov and G. V. Khodova, Phys. Stat. Sol. B 150, 545 (1988); N. N. Rosanov, Spatial Hysteresis and Optical Patterns (Springer, Berlin, 2002)

[4] M. Tlidi, P. Mandel and R. Lefever, Phys. Rev. Lett. 73, 640 (1994)

[5] W. J. Firth and A. J. Scroggie, Phys. Rev. Lett. 76, 1623 (1996)

[6] T. Ackemann, W. J. Firth and G.-L. Oppo, Adv. Atom. Mol. Opt. Phys. 57, 323 (2009)

[7] P. Grelu and N. Akhmediev, Nature Photonics 6, 84 (2012)

[8] G.-L. Oppo, M. Brambilla and L. A. Lugiato, Phys. Rev. A 49, 2028 (1994)

[9] G. J. de Valcarcel, K. Staliunas, E. Roldan and V. J. Sanchez-Morcillo, Phys. Rev. A 54, 1609 (1996)

[10] C. Etrich, U. Peschel and F. Lederer, Phys. Rev. E 56, 4803 (1997)

[11] P. Lodahl and M. Saffman, Phys. Rev. A 60, 3251 (1999)

[12] S. Ducci, N. Treps, A. Maitre and C. Fabre, Phys. Rev. A 64, 023803 (2001).

[13] M. Shelton and D. P. West, Opt. Express 9, 16 (2001).

[14] M. Peckus, K. Staliunas, M. Saffman, G. Slekys, V. Sirutkaitis, V. Smilgevicius and R. Grigonis, Opt. Commun. 251, 165 (2005).

[15] M. Peckus, K. Staliunas, Z. Nizauskaite and V. Sirutkaitis, Opt. Lett. 32, 3014 (2007)

[16] G.-L. Oppo, M. Brambilla, D. Camesasca, A. Gatti and L. A. Lugiato, J. Mod. Opt. 41, 1151 (1994)
[17] G.-L. Oppo, A. J. Scroggie and W. J. Firth, Phys. Rev. E 63, 066209 (2001)

[18] K. Staliunas, J. Mod. Opt. 42, 1261 (1995)

[19] S. Longhi and A. Geraci, Phys. Rev. A 54, 4581 (1996)

[20] V. J. Sanchez-Morcillo, E. Roldan, G. J. de Valcarcel and K. Staliunas Phys. Rev. A 56, 3237 (1997)

[21] M. Santagiustina, E. Hernandez-Garcia, M. San-Miguel, A. J. Scroggie and G.-L. Oppo, Phys. Rev. E 65, 036610 (2002)

[22] S. T. Yang, R. C. Eckardt and R. L. Byer, Opt. Lett. 18, $971(1993)$

[23] W. R. Bosenberg, A, Drobshoff, J. I. Alexander, L. E. Meyers and R. L. Byer, Opt. Lett. 21, 1336 (1996)

[24] M. E. Klein, C. K. Laue, D.-H. Lee, K.-J. Boller and R. Wallenstein, Opt. Lett. 25, 490 (2000)

[25] A. Henderson and R. Stafford, Opt. Express 14, 767 (2006)

[26] L. B. Kreuzer, in Proceedings of the Joint Conference on Lasers and Opto-Electronics (Institution of Electrical and Radio Engineers, London, 1969), page 52

[27] W. Brunner, R. Fischer and H. Paul, Ann. Phys. (Leipzig) 30, 299 (1973).

[28] W. Brunner and H. Paul, Prog. Opt. 15, 1 (1977).

[29] E. Rosencher and C. Fabre, J. Opt. Soc. Am. B 19, 1107 (2002)

[30] C. R. Phillips and M. M. Fejer, J. Opt. Soc. Am. B 27, $2687(2010)$

[31] G.-L. Oppo, J. Math. Chem. 45, 95 (2009)

[32] R. W. Boyd, Nonlinear Optics, (Academic Press, New York, 2008)

[33] D. Cuozzo and G.-L.Oppo, Phys. Rev. A 84, 043810 (2011) 
[34] F. Prati, G. Tissoni, C. McIntyre and G.-L. Oppo, Eur. Phys. J. D 59, 139 (2010)

[35] S. Ciliberto, P. Coullet, J. Lega, E. Pampaloni and C. Perez-Garcia, Phys. Rev. Lett. 65, 2370 (1990)

[36] P. Coullet and K. Emilsson, Physica A 188, 190 (1992)

[37] P. Coullet and K. Emilsson, Physica D 61, 119 (1992)

[38] G.-L. Oppo, A. Politi, G. L. Lippi and F. T. Arecchi, Phys. Rev. A 34, 4000 (1986)

[39] Q. Ouyang and H. L. Swinney, Chaos 1, 411 (1991)

[40] G. K. Harkness, J. C. Lega and G.-L. Oppo, Chaos, Solitons and Fractals 4, 1519 (1994)

[41] C. Bonatto, M. Feyereisen, S. Barland, M. Giudici, C. Masoller, J. R. Rios Leite and J. R. Tredicce, Phys. Rev. Lett. 107, 053901 (2011); J. Zamora-Munt, B. Garbin, S. Barland, M. Giudici, J. R. Rios Leite, C. Masoller and J. R. Tredicce, Phys. Rev. A 87, 035802 (2013).
[42] S. Longhi, Phys. Scr. 56, 611 (1997)

[43] K. Staliunas and V. J. Sanchez-Morcillo Opt. Comm. 139, 306 (1997)

[44] G.-L. Oppo, A. J. Scroggie and W. J. Firth J. Opt. B 1, $133(1999)$

[45] S. Longhi, Opt. Comm. 149, 335 (1998)

[46] G. J. de Valcarcel, E. Roldan and K. Staliunas Opt. Comm. 181, 207 (2000)

[47] K. Staliunas and V. J. Sanchez-Morcillo, arXiv:nlin /0002029 (2000)

[48] J. M. McSloy, W. J. Firth, G. K. Harkness and G.-L. Oppo, Phys. Rev. E 66, 046606 (2002)

[49] D. J. B. Lloyd, B. Sandstede, D. Avitabile and A. R. Champneys, SIAM J. App. Dyn. Syst. 7, 1049 (2008) 